\title{
CERTAIN CLASS-NUMBER RELATIONS IMPLIED IN THE NACHLASS OF GAUSS*
}

\author{
BY E. T. BELL
}

1. Introduction. In a previous note $\dagger$ it was pointed out that the fundamental expansions of the elliptic theta constant products of the third degree, due to Hermite and Kronecker, follow by a mere change of sign in an algebraic identity from sections 292-3 of the Disquisitiones Arithmeticae of Gauss. The posthumous notes of Gauss, on what would now be called the transformation of the elliptic theta functions, contain implicitly a great many further classnumber relations of types much more abstruse than the famous eight of Kronecker. To justify this assertion we need translate only two of the simplest of Gauss' identities into their equivalents in terms of class numbers. These will be sufficient; several of the remaining identities give classnumber relations of equal simplicity and elegance. The present relations are allied to some that were stated without any clue to their origin by Liouville, $\ddagger$ also to others deduced by Gierster $\S$ from the theory of modular correspondences. It is surprising that these simple and extremely elegant relations should have been overlooked, lying as they do on the very surface of Gauss' work. All are written down by the usual device of comparing coefficients in different expansions of the same functions. It will not be necessary to preserve the algebraic details beyond the statement of the relevant identities (those given by Gauss for the transformation of the third order), as all the technique is standard. The final formulas (1)-(18) have been verified numerically.

*Presented to the Society, San Francisco Section, April 7, 1928.

$\uparrow$ This Bulletin, vol. 30 (1924), pp. 236-8.

$\ddagger$ For references, see Dickson's History, vol. 3, Chap. 6; also H. J. S. Smith, Report on the Theory of Numbers.

§Mathematische Annalen, vol. 21 (1883), p. 49. 
2. The Functions $A, B, C, H, K$. If $\mu$ is an odd integer, we shall write $(-1 \mid \mu) \equiv(-1)^{(\mu-1) / 2}$, so that $(-1 \mid-\mu)=$ $-(-1 \mid \mu)$. The functions $A, \cdots, K$ are defined as follows, where $m$ is an odd integer $>0$, and in each case the $\sum$ refers to all $\mu$ such that, for $m$ constant, the indicated (indeterminate) equation is satisfied; $\nu$ denotes an arbitrary (odd or even) integer $\gtreqless 0$, and $\mu, \mu_{1}$ are odd integers $\gtreqless 0$.

$$
\begin{aligned}
m & =16 \nu+3 \mu^{2}: & A(m) & =\sum(-1 \mid \mu) \mu . \\
m & =4 \mu_{1}^{2}+3 \mu^{2}: & B(m) & =\sum(-1 \mid \mu) \mu . \\
4 m & =\mu_{1}{ }^{2}+3 \mu^{2}: & C(4 m) & =\sum(-1 \mid \mu) \mu . \\
m & =12 \nu^{2}+\mu^{2}: & H(m) & =\sum(-1 \mid \mu) \mu . \\
4 m & =3 \mu_{1}^{2}+\mu^{2}: & K(4 m) & =\sum(-1 \mid \mu) \mu .
\end{aligned}
$$

For example, if $m=19$, the values of $(\nu, \mu)$ from which $A(m)$ is computed are $(\nu, \mu)=( \pm 1, \pm 1)$, and hence $A(19)=4$; for $m=27$ we have $(\nu, \mu)(0, \pm 3)$, and hence $A(27)=-6$.

For these we find the following theorems.

$$
\begin{aligned}
m & \equiv 3 \bmod 8: \\
6 A(m) & =3 C(4 m)=2 H(3 m)=K(12 m) . \\
m & \equiv 11 \bmod 24: \\
A(m) & =C(4 m)=H(3 m)=K(12 m)=0 . \\
m & \equiv 23 \bmod 24: \\
B(m) & =K(12 m)=H(3 m)=0 . \\
m & \equiv 5 \bmod 6: C(4 m)=0 . \\
m & \equiv 7 \bmod 8: \\
2 H(3 m) & =K(12 m)=-6 B(m) . \\
m & \equiv 3,19 \bmod 24: 6 A(m)=-K(4 m) .
\end{aligned}
$$

Certain of these can be seen by inspection, on noting what numbers are not represented in the given forms; all, however, follow at once by the means indicated in $\$ 4$.

3. Class-Number Relations. For uniformity we shall express all the relations in terms of $E$ alone. The notation 
is that of H. J. S. Smith's Report on the Theory of Numbers; it agrees with Dickson's History (vol. 3, chap. 6); $F(n)$, $F_{1}(n)$ are respectively the numbers of odd, even classes for the negative determinant $-n ; E(n) \equiv F(n)-F_{1}(n)$, so that $12 E(n)$ is the total number of representations of $n$ as a sum of three squares. By convention $F(0)=0, E(0)=1 / 12$, and a class equivalent to $a\left(x^{2}+y^{2}\right)$ ( $a \equiv$ an integer) contributes $1 / 2$ to $F$ or to $F_{1}$, while a class equivalent to $a\left(2 x^{2}+2 x y\right.$ $+2 y^{2}$ ) contributes $1 / 3$ to $F_{1}$. For expressing $E$ in terms of $F$ and $F_{1}$ there are the following well known relations, $(n \geqq 0)$,

$$
\begin{gathered}
3 E(8 n+3)=2 F(8 n+3), E(8 n+7)=0, E(4 n)=E(n), \\
E(4 n+1)=F(4 n+1), E(4 n+2)=F(4 n+2), F(4 n)=2 F(n) .
\end{gathered}
$$

A convenient table for numerical verifications was given in a former paper.*

We shall call two relations independent if neither can be inferred from the other either by the theorems (1)-(6) of $\$ 2$, or by the relations between $E, F, F_{1}$ stated above. The sense in which class-number relations are $n$-tuply infinite, $n>0$, was defined and amply illustrated elsewhere $\nmid$; briefly, a class-number relation involving arbitrary uniform functions, or arbitrary odd or even uniform functions of precisely $n$ independent variables, was called $n$-fold infinite; if no arbitrary function occurs in the relation, (we say that) the relation is constant. All the class-number relations reported in Dickson's History (loc. cit.) are constant in this sense; they all are degenerate cases of singly infinite relations. The relations next stated are equivalent to all those constant relations deducible from the transformation of the third order of the elliptic theta functions, combined with the transformation of the first order, and hence the corresponding Gauss formulas can give nothing further. It is to be particularly noted that we have included in this statement the transformations of the first order, as otherwise

* Tôhoku Mathematical Journal, vol. 19 (1921), p. 116.

† Quarterly Journal of Mathematics, vol. 49 (1923), pp. 322-337. 
certain of the formulas may not be evident. The relations are not all independent in the sense above defined; we have given the dependent ones on account of their relative simplicity. The set is as follows. The $\sum$ refers to all integers $\nu \gtreqless 0$, or to all odd integers $\mu \gtrless 0$, such that the arguments of the summands are $\geqq 0$.

(7) $m \equiv 11 \bmod 24:$

$$
\sum\left[E\left(m-48 \nu^{2}\right)-E\left(m-3 \nu^{2}\right)\right]=0 .
$$

(8) $m \equiv 5 \bmod 6$ :

$$
\sum(-1)^{\nu} E\left(m-3 \nu^{2}\right)=0 \text {. }
$$

(9) $m \equiv 23 \bmod 24$ :

$$
\sum\left[E\left(m-12 \mu^{2}\right)-E\left(m-3 \mu^{2}\right)\right]=0 .
$$

(10) $m \equiv 3,19 \bmod 24$ :

$$
4 \sum\left[E\left(m-48 \nu^{2}\right)-E\left(m-3 \mu^{2}\right)\right]=A(m) \text {. }
$$

(11) $m \equiv 1,3 \bmod 6$ :

$$
8 \sum(-1)^{\nu} E\left(m-3 \nu^{2}\right)=C(4 m) \text {. }
$$

(12) $m \equiv 7,15 \bmod 24$ :

$$
4 \sum\left[E\left(m-12 \mu^{2}\right)-E\left(m-3 \mu^{2}\right)\right]=-B(m) \text {. }
$$

(13) $m \equiv 1 \bmod 24$ :

$$
\begin{gathered}
12 \sum\left[E\left(\frac{m-\mu^{2}}{12}\right)-E\left(\frac{m-4 \nu^{2}}{3}\right)\right]=H(m), \\
\mu \equiv 1,5 \bmod 6, \quad \nu \equiv 2,4 \bmod 6 ;
\end{gathered}
$$

namely, the $\sum$ refers only to such odd $\mu \gtrless 0$, and to such arbitrary $\nu \gtreqless 0$, as indicated, and similarly in (14), (17).

(14) $m \equiv 13 \bmod 24$ :

$$
\begin{aligned}
& 12 \sum\left[E\left(\frac{m-\mu^{2}}{12}\right)-E\left(\frac{m-4 \nu^{2}}{3}\right)\right]=-H(m), \\
& \mu \equiv 1,5 \bmod 6, \quad \nu \equiv 1,5 \bmod 6 .
\end{aligned}
$$

(15) $m \equiv 3 \bmod 8$ :

$$
12 \sum\left[E\left(\frac{m-3 \mu^{2}}{4}\right)-E\left(m-48 \nu^{2}\right)\right]=H(3 m) \text {. }
$$


(16) $m \equiv 7 \bmod 8$ :

$$
12 \sum\left[E\left(\frac{m-3 \mu^{2}}{4}\right)-E\left(m-12 \mu^{2}\right)\right]=-H(3 m) \text {. }
$$

(17) $m \equiv 1 \bmod 6$ :

$$
24 \sum\left[E\left(\frac{m-\mu^{2}}{3}\right)-E\left(\frac{m-4 \nu^{2}}{3}\right)\right]=K(4 m),
$$

$\mu \equiv 1,5 \bmod 6, \quad \nu \equiv 1,2 \bmod 3$.

(18) $m \equiv 7 \bmod 8$ :

$$
24 \sum\left[E\left(m-3 \mu^{2}\right)-E\left(m-12 \mu^{2}\right)\right]=K(12 m) .
$$

4. Proofs. As remarked in $\$ 1$, it is unnecessary to go into details, although some of the formulas in $\$ \$ 2-3$ follow only after several reductions. It is essential however to state from what identities of Gauss all of the preceding follow as indicated. The requisite relations are given (in a slightly different notation) by Gauss (Werke, vol. 3, p. 471), and are

$$
3 P p=\frac{r^{3}}{R}-\frac{q^{3}}{Q}, \quad P p=\frac{Q^{3}}{q}-\frac{R^{3}}{r}
$$

where, for $|x|<1$,

$$
p \equiv \sum x^{\nu^{2}}, \quad q \equiv \sum(-1)^{\nu} x^{\nu^{2}}, \quad r \equiv \sum x^{\mu / 4},
$$

the $\sum$ extending to all integers $\nu \gtreqless 0$, or to all odd integers $\mu \gtrless 0$, and $P, Q, R$ are obtained from $p, q, r$ respectively on replacing $x$ by $x^{3}$. Independent proofs of Gauss' identities from the theta formula of Schröter have been given by Goring.*

California Institute of Technology

*Mathematische Annalen, vol. 7 (1874), pp. 325 et seq. 